\title{
Propuestas de regulación frente a una nueva brecha digital por razón de género: ciberviolencia contra la mujer a la luz del marco europeo de protección de datos*
}

Regulatory proposals vis-à-vis a new digital gender equality gap: cyberviolence against women in light of the European data protection framework

\author{
MÓNICA MARTÍNEZ LÓPEZ-SÁEZ \\ Investigadora García Pelayo \\ Centro de Estudios Políticos y Constitucionales (España) \\ monica.martinez@cepc.es \\ https://orcid.org/0000-0002-6111-5798
}

Resumen: Las investigaciones realizadas hasta la fecha confirman que las mujeres y niñas son las principales víctimas de las manifestaciones más graves de la violencia facilitada por las NTIC. Por ello a la desafortunadamente "clásica" desigualdad entre hombres y mujeres tenemos que añadir lo que hemos llamado en este trabajo como "brecha digital por razón de género", entendida como el traslado al ámbito digital de las desigualdades, estereotipos y actos de violencia que sufren las mujeres, por el mero hecho de ser mujer. Entre las diversas conductas que incluye, en el presente trabajo se han examinado dos casos concretos de ciberviolencia (en concreto dos casos de violencia digital sexualizada) contra la mujer desde la perspectiva de la protección de datos y a la luz del principio favor libertatis. En el contexto del tratamiento de datos personales, son numerosos los supuestos habilitantes para reforzar la protección de las mujeres como principales víctimas de este tipo de violencia con independencia de que la conducta llevada a cabo sea o no un ilícito penal y sobre todo teniendo en cuenta los obstáculos o retrasos en poner en marcha

Cómo citar este trabajo: MARTÍNEZ LÓPEZ-SÁEZ, Mónica, "Propuestas de regulación frente a una nueva brecha digital por razón de género: ciberviolencia contra la mujer a la luz del marco europeo de protección de datos", Revista de Estudios Jurídicos y Criminológicos, n. ${ }^{\circ} 4$, Universidad de Cádiz, 2021, pp. 211-233, DOI: https://doi.org/10.25267/REJUCRIM.2021.i.4.08

* Esta aportación se ha elaborado en el marco del proyecto de investigación AICO/2019/205 (Conselleria de Educación, Investigación, Cultura y Deporte de la Comunitat Valenciana, IP Beatriz Tomás Mallén) y del proyecto de investigación RTI2018-095367-B-I00 (Ministerio de Ciencia, Innovación y Universidades, IP Rosario García Mahamut y Cristina Pauner Chulvi) ambos relativos a la implementación del RGPD en España. 
mecanismos efectivos en sede policial y judicial. Analizamos los principios de protección de datos y proponemos diferentes vertientes del derecho al olvido no sólo como garantías de una autodeterminación informativa y de un empoderamiento femenino, sino también en una garantía necesaria para la igualdad efectiva y para la recuperación del trauma más frecuente de las mujeres del siglo XXI.

\begin{abstract}
Research to date confirms that women and girls are the main victims of the most serious displays of NICT-facilitated violence. Therefore, to the unfortunately-common inequality between men and women we have to add what we have called the "gender digital divide", understood as the transfer of inequalities, stereotypes and violent acts suffered by women (simply because they are women) to the digital sphere. Among the various behaviors included, this paper has dealt with two specific cases of cyber-violence (specifically two cases of sexualized digital violence) against women from a data protection perspective and in light of the favor libertatis principle. In the context of personal data processing, there are numerous scenarios that allow for strengthened protections for women regardless of whether or not the conduct carried out is a criminal offense and especially taking into account the obstacles or delays in putting in place effective mechanisms in the police and judicial context. We analyze the various data protection principles and propose different gradations of the right to be forgotten, not only as a guarantee of informational self-determination and female empowerment, but also as a necessary guarantee for effective equality and for the recovery of the most frequent trauma of $21^{\text {st }}$ century women.
\end{abstract}

Palabras claves: ciberviolencia contra la mujer, brecha digital, perspectiva de género, protección de datos, principio favor libertatis.

Keywords: cyberviolence again women, digital divide, gender perspective, data protection, favor libertatis principle.

Sumario: 1. INTRODUCCIÓN. 2. LA NECESARIA PERSPECTIVA DE GÉNERO ANTE LA TRANSFORMACIÓN SOCIO-DIGITAL. 3. APROXIMACIÓN A LA VIOLENCIA DE GÉNERO Y A DOS CASOS DE CIBERVIOLENCIA CONTRA LA MUJER DESDE EL ÁMBITO DE LA PROTECCIÓN DE DATOS. 4. EL 'DERECHO AL OLVIDO' COMO GARANTÍA JURÍDICA Y DIGITAL: PROPUESTAS DE FERENDA Y LEGE DATA PARA ASEGURAR LA DIGNIDAD DE LAS MUJERES VÍCTIMAS DE CIBERVIOLENCIA SEXUALIZADA. 5. CONCLUSIÓN. 6. BIBLIOGRAFÍA.

\title{
1. INTRODUCCIÓN
}

La persona está sujeta a una "lotería" bio-social". Ante esta "paradoja de la vulnerabilidad" observamos su vertiente universal y particular: en tanto seres corporales y sintientes, todas somos vulnerables o susceptibles a daños, pero experimentamos esa vulnerabilidad de manera singular y única en función de nuestro cuerpo, mente y rol

\footnotetext{
${ }^{1}$ MASFERRER DOMINGO, A., "Vulnerability and Dignity in the Age of Rights", en AA. VV., Human Dignity of the Vulnerable in the Age of Rights. Interdisciplinary Perspectives, Basel, Springer, 2016, p. 12.
} 
dentro del entramado socio-económico-institucional ${ }^{2}$. Si bien algunas de las causas de vulnerabilidad son temporales y superables, otras son permanentes y ancladas en construcciones socio-culturales más difíciles de revertir. La prevalencia de las mujeres como víctimas de violencia, en general, y de violencia digital, en particular, es un claro ejemplo de ello, produciéndose, en parte, como resultado de las construcciones socioculturales sobre el sexo y los roles y actitudes asociados al mismo. La igualdad entre hombres y mujeres en la que se basan todos los ordenamientos democráticos, sociales y basados en el Derecho, hace inadmisible, a priori, calificar a las mujeres como personas vulnerables, en tanto en cuanto la vulnerabilidad no es inherente a la condición femenina perse.

Sin embargo, las relaciones sociales no existen en un vacío espacio-temporal, sino en tiempos y espacios socio-culturales que sí que muestran desigualdades o estructuras que potencian resultados desfavorables en función de (o basados en) factores perceptibles y determinantes, incluido el sexo. El Derecho, como encargado de regularlas, también estará sujeto a los mismos, y, por ende, deberá adaptarse y reinventarse ante los cambios sociales que vaya surgiendo. En esta línea, se ha utilizado la categorización o clasificación del sexo femenino no sólo como criterio de discriminación (el llamado "motivo protegido"), sino también como grupo vulnerable con el único fin de asegurar y garantizar la protección efectiva de los derechos que son universales en la teoría, pero que, en la práctica, se observa que las mujeres se ven envueltas en determinadas situaciones, directa o indirectamente por razón de su sexo, y ello las coloca en situaciones discriminatorias o de riesgo, y por ende, de vulnerabilidad.

Por ello, y sin ánimo de menospreciar la situación sanitaria en la que nos encontramos inmersos, y en palabras de la Organización Mundial de la Salud (en adelante, la OMS), se puede calificar la violencia contra las mujeres como un problema de "salud pública mundial de proporciones epidémicas" 3 . La violencia contra la mujer, mundialmente conocida como violencia de género, no deja de ser un "símbolo brutal de la desigualdad" y una "clara manifestación de discriminación"4. Los estudios confirman ya no sólo las desigualdades entre hombres y mujeres en ámbitos como el laboral, sino que también confirman los atentos contra la salud, la seguridad y la autonomía de las mujeres y niñas ${ }^{5}$.

\footnotetext{
2 PERONI, L. y TIMMER, A., "Vulnerable groups: The promise of an emerging concept in European Human Rights Convention Law”, International Journal of Constitutional Law, Vol. 11, núm. 4, 2013, pp. 1058-1059.

${ }^{3} \mathrm{OMS}$, Resumen de orientación Estimaciones mundiales y regionales de la violencia contra la mujer: prevalencia y efectos de la violencia conyugal y de la violencia sexual no conyugal en la salud, OMS, Ginebra, 2013,p. 1.

${ }^{4}$ RIDAURA MARTÍNEZ, M.J., "El sentido actual de la Ley Orgánica de Medidas de Protección Integral contra la Violencia de Género", Estudio Integral de la Violencia de Género: un análisis teórico-práctico desde el Derecho y las Ciencias Sociales, (dir. Martín, M.), Valencia, Tirant lo Blanch, 2018, p. 138.
}

5 AGENCIA DE LOS DERECHOS FUNDAMENTALES DE LA UE, Violencia de género contra las mujeres: una encuesta a escala de la UE. Resumen de las conclusiones, Oficina de Publicaciones de la Unión Europea, Luxemburgo, 2014. 
El problema añadido al que nos enfrentamos, en la actualidad, es que este 'virus' social se ha expandido y ha penetrado el reino virtual; confirmando las investigaciones realizadas hasta la fecha que las mujeres y niñas son las principales víctimas de las manifestaciones más graves de la violencia facilitada por las Nuevas Tecnologías de la Información y la Comunicación (en adelante, las NTIC) ${ }^{6}$. De tal forma que la violencia de género, incluida la discriminación, el acoso y agresión sexual o emocional, etc., ahora también se comete, se facilita y se propaga en el mundo virtual: "el fenómeno de las redes sociales y la mensajería instantánea provocará [...] una amplificación de las conductas coercitivas, una extensión del control y la manipulación, así como la persistencia y [...] presencia del agresor [...] que le produce a la víctima una sensación de desamparo e impotencia ante los hechos [...] que está sufriendo [...] una grave alteración en su salud fisica y mental y [...] su vida cotidiana"7.

Las NTIC no sólo tienen el poder y potencial de conectar a personas, abrir mentes y ser catalizadores de sendos progresos y revoluciones socio-culturales, sino también de reforzar y difundir determinadas estructuras que impactan negativamente a ciertos colectivos, incluidas las mujeres. La revolución tecnológica y la transformación digital han traído no sólo nuevas maneras de relacionarse con los demás o nuevas formas de comunicación social hacia la igualdad entre hombres y mujeres, sino también nuevas formas de atacar y silenciar a las mujeres ${ }^{8}$. Los comportamientos y fenómenos sociales dañinos que observamos en nuestro día a día se vierten en la Red, y esta, por su propia naturaleza, los intensifica y agrava su alcance y consecuencias. Esta nueva realidad, llamada por algunos como "ciberviolencia de género", es solo una manifestación más del paradigma socio-cultural existente trasladado a la cultura online, convirtiéndose la Red en herramienta para la opresión y abuso hacia las mujeres. Además, la violencia contra las mujeres debe interpretarse como un continuum, pues las diferentes formas de violencia en muchas ocasiones están interconectadas y no necesariamente son excluyentes entre sí $^{10}$.

Con todo lo anterior, el presente trabajo justifica la necesidad de adoptar una pesrpectiva de género ante los nuevos fenómenos socio-digitales, propone una nueva definición de brecha digital por razón de género y analiza el marco europeo de protección de datos con

\footnotetext{
${ }^{6}$ INSTITUTO EUROPEO DE IGUALDAD DE GÉNERO, Cyber violence against women and girls, Oficina de Publicaciones de la Unión Europea, Luxemburgo, 2017, p. 3.

${ }^{7}$ Vid., SALA ORDOÑEZ, R., "L'estat embrionari de la violència de gènere digital", Món Jurídic: Revista de l'il'Lustre Col-Legi de l'advocacia de Barcelona, núm. 323 (junio-julio), 2019, pp. 20-21.

${ }^{8}$ HARRIS, B., "Spacelessness, Spatiality and Intimate Partner Violence: Technology-facilitated Abuse, Stalking and Justice", en AA. VV. Intimate Partner Violence, Risk and Security: Securing Women's Lives in a Global World, Nueva York y Oxon, Routledge, 2018, p. 53.

${ }^{9}$ QUESADA AGUAYO, M.S., "La violencia de género y el ciberacoso en las redes sociales: análisis y herramientas de detección", Ciberacoso y violencia de género en redes sociales: Análisis y herramientas de prevención (coord. Verdejo, M.A.), Sevilla, Universidad Internacional de Andalucía, 2015, p. 148.

${ }^{10}$ POWELL, A., y HENRY, N., Sexual Violence in a Digital Age, Melbourne, Palgrave MacMillan, 2017, p. 154.
} 
el fin de realizar unas consideraciones y propuestas hermenéuticas sobre las garantías al alcance de las mujeres como principales víctimas de ciberviolencia.

\section{LA NECESARIA PERSPECTIVA DE GÉNERO ANTE LA TRANSFORMACIÓN SOCIO-DIGITAL}

Se ha prestado considerable atención a comportamientos violentos que se perpetran con la ayuda de las NTIC, en general, y aquellos relativos a colectivos vulnerables, en particular, sobre todo en relación con la explotación infantil en Internet o el ciberacoso a menores ${ }^{11}$. No obstante, resulta necesario centrar nuestra atención, cuando hablamos de la aparición de nuevas formas de "violencia digital" 12 , en aquella ejercida mediante la divulgación de datos de carácter personal desde la perspectiva de género. La necesidad de estudiar la violencia digital desde esta perspectiva concreta de género tiene su fundamento en dos razones principales.

De una parte, las investigaciones realizadas hasta la fecha confirman que las mujeres y niñas son las principales víctimas de las manifestaciones más graves de la violencia digital ${ }^{13}$. Al igual que a nivel mundial, la violencia de género en Europa afecta de manera desproporcionada a las mujeres (una de cada tres mujeres europeas experimenta violencia física o sexual a partir de su decimoquinto cumpleaños) ${ }^{14}$, revelando patrones desiguales y discriminatorios, y comprende no sólo el ejercicio de actos físicos o psicológicos presenciales sino también aquellos actos que tienen cabida a través de las NTIC. Estas

\footnotetext{
${ }^{11}$ A modo de ejemplo, vid. MONTIEL JUAN, I., "Cibercriminalidad social juvenil: la cifra negra”, Revista de los Estudios de Derecho y Ciencia Política (IDP), núm. 22, 2016, pp. 119-131.

${ }^{12}$ Así lo llama, por ejemplo, la AEPD en sus Recomendaciones "Ayuda a las víctimas de violencia de género y violencia digital”. Disponible en: https:/www.aepd.es/es/areas-de-actuacion/recomendaciones

${ }^{13}$ FLORES FERNÁNDEZ, J., "Privacidad, factor de riesgo y protección en la violencia digital contra las mujeres", Ciberacoso y violencia de género en redes sociales: Análisis y herramientas de prevención (coord. Verdejo, M.A.), Sevilla, Universidad Internacional de Andalucía, 2015, p.313; VILLANUEVABLASCO, V.J. y SERRANO-BERNAL, S., "Patrón de uso de internet y control parental de redes sociales como predictor de sexting en adolescentes: una perspectiva de género", Revista de Psicología y Educación, Vol. 14, núm. 1, 2019, p. 23; todo ello confirmado con estadísticas mundiales: vid., OCDE, Report: Bridging The Digital Gender Divide. Include. Upskill. Innovate, OCDE, 2018, p. 23. (https://www.oecd.org/digital/bridging-the-digital-gender-divide.pdf, consulta: 06/05/2021).

14 AGENCIA DE LOS DERECHOS FUNDAMENTALES DE LA UE, Violencia de género contra las mujeres: una encuesta a escala de la UE. Resumen de las conclusiones, Oficina de Publicaciones de la Unión Europea, Luxemburgo, 2014, pp. 3, 9, y 13. Aunque el estudio se centró en violencia ejercida por parte de quien es o haya sido cónyuge, o que esté o haya estado ligado a ella por relaciones similares de afectividad, aun sin convivencia), igualmente permite afirmar que las cifras, de violencia de género, en general, y la ejercida mediante las NTIC, en particular, son mucho más altas en la práctica (pues existen numerosos casos de violencia contra las mujeres ejercida por parte de aquellos que no tienen ni han tenido una relación sentimental). Además, según el Instituto Europeo de la Igualdad de Género, la mayoría de los casos de violencia de género (digital o no), no se denuncian, confirmando que, si bien no se han realizado encuestas desglosadas por género a nivel europeo y la investigación es limitada a nivel nacional, la que hay disponible "sugiere que las mujeres son desproporcionadamente objetivos de ciertas formas de violencia cibernética en comparación con los hombres". Vid. INSTITUTO EUROPEO DE IGUALDAD DE GÉNERO, Cyber violence against women and girls, Oficina de Publicaciones de la Unión Europea, Luxemburgo, 2017, p. 3 (https://eige.europa.eu/publications/cyber-violence-against-women-and-girls, consulta: 06/05/2021).
} 
manifestaciones en su mayoría pueden ser clasificadas como una suerte de violencia digital sexualizada ${ }^{15}$, con ejemplos que van desde el acoso sexual en línea, la sextorsión a las agresiones sexuales facilitadas por aplicaciones de concertación de citas ${ }^{16}, \mathrm{y}$, por ende, revelan que el fenómeno no parece ser neutral por lo que concierne al género.

De otra parte, tanto las causas como las consecuencias de este fenómeno se deben, en gran medida, a expectativas obsoletas, estereotipos y prejuicios de lo que es masculino y femenino ${ }^{17}$, a la normalización de la hipersexualización y cosificación de la mujer, y, por tanto, a una jerarquización socio-cultural que ha creado relaciones (inequitativas) de poder entre hombres y mujeres ${ }^{18}$.

Por establecer una definición operativa de violencia contra las mujeres, se podría definir como una como una categoría concreta de violencia de género (patrones y "conductas violentas y coercitivas" 19 dirigidas contra una persona por motivo de su género/sexo), es decir, como aquella violencia dirigida contra la mujer, por el mero hecho de serlo, y que resulta o puede resultar en un daño físico, psicológico, económico, o, cualquier otro tipo de sufrimiento. Si bien no existe una definición común ni universalmente reconocida, las definiciones, tanto en el ámbito internacional como europeo, presentan notables similitudes ${ }^{20}$. Con independencia de los matices de su conceptualización, la violencia contra la mujer indudablemente constituye una discriminación y una violación generalizada del disfrute de los derechos más fundamentales de este colectivo.

Lo cierto es que la innovación y transformación tecno-digital, sobre todo en lo que al acceso instantáneo y constante a la información, comunicación o incluso a la geolocalización, se refiere, han creado oportunidades singulares para perpetrar actos precursores o propios de este tipo de violencia. Además, la velocidad y universalidad en la difusión, acceso y perdurabilidad de contenidos sensibles y datos personales ha otorgado a los maleantes una gran facilidad para poner en entredicho la seguridad e

\footnotetext{
${ }^{15}$ ESTEBÁNEZ, I. y VÁZQUEZ, N., La desigualdad de género y el sexismo en las redes sociales. Una aproximación cualitativa al uso que hacen de las redes sociales las y los jóvenes de la CAPV, VitoriaGasteiz, Servicio Central de Publicaciones del Gobierno Vasco, 2013, pp. 95-97; FERREIRO, V., VILÀ, R., y PRADO, N., "Experiencias sexistas en las redes sociales. Perpetuando la violencia de género", Psicología y Educación: Presente y Futuro (coord. Castejón, J.L.), Alicante, ACIPE, 2016, pp. 588-589.

${ }^{16}$ Vid., por todas las noticias, SANDELL, S., "Dating Apps, Rape and the Lack of Safety in Online Dating", HuffPost Blog, 24-02-2016 (revelando que el 40\% de las agresiones sexuales se cometen con ayuda de las NTIC y aplicaciones de citas y que más del $85 \%$ de las víctimas son mujeres).

17 Vid., QUESADA AGUAYO, M.S., "Género y discriminaciones asociadas al hecho de ser mujer", Manual Agentes de Igualdad (coord. Román, M.), Sevilla, Diputación de Sevilla, 2009, pp. 30-42.

${ }^{18}$ QUESADA, M.S., "La violencia de género...op. cit., p. 133.

19 De hecho, se ha definido directamente desde la perspectiva del colectivo femenino. Vid., SALA ORDOÑEZ, R., “L'estat embrionari de la la violència de gènere...op.cit., p. 20.

${ }^{20}$ A nivel europeo (entendido en su sentido más amplio), se ha definido como los "actos de violencia basados en el género que implican o pueden implicar para las mujeres daños o sufrimientos de naturaleza física, sexual, psicológica o económica, incluidas las amenazas de realizar dichos actos, la coacción o la privación arbitraria de libertad, en la vida pública o privada”. Vid. Art. 3(a) del Convenio de Estambul del Consejo de Europa.
} 
intimidad de las mujeres, y todo ello mediante la vigilancia constante y conductas dañinas a distancia. Lo que resulta de todo punto innegable es que la propia naturaleza de las NTIC ha dado lugar a nuevos riesgos para la intimidad, integridad física y moral, y, en última instancia, riesgos para la dignidad y el libre desarrollo de este grupo en situación de especial vulnerabilidad; no sólo por la situación de desprotección por diseño ${ }^{21}$ en la que se encuentran, sino también, y sobre todo, por la incapacidad para defenderse frente a dichas injerencias. Con todo lo anterior, se puede apreciar cómo la Red ha dejado de ser un entorno neutral, no porque no se esté luchando por ello, ni porque la tecnología no tenga la capacidad o esté diseñada para serlo (aunque esto también es debatible ${ }^{22}$ ), sino porque el mundo virtual es un reflejo del mundo real ${ }^{23}$, y este está plagado de desigualdades.

De tal forma que a la desafortunadamente "clásica" desigualdad entre hombres y mujeres tenemos que añadirle la llamada "brecha digital por razón de género" (digital gender equality gap). Aunque gran parte de organismos internacionales han enfocado su definición en lo que se refiere a la falta de acceso, competencias digitales, utilización de las NTIC o la falta de presencia en mercados laborales tecnológicos o industrializados por parte del colectivo femenino, en el presente trabajo, 'brecha digital por razón de género' nos referimos al traslado de las desigualdades, estereotipos y actos de violencia que sufren las mujeres, por el mero hecho de ser mujer, al ámbito digital ${ }^{24}$. Esta nueva brecha digital por razón de género incluye conductas de diversa índole: desde insultos sexistas (slut-shaming) o expresiones de odio e incitación a la violencia (gender-based hate speech), al sexting (como resultado de la presión social de las mujeres y niñas por parte de compañeros sentimentales masculinos) y la sextorsión, a prácticas que se acercan más al acoso tales como el "cibercontrol" (vigilancia continuada de la actividad online u offline pero apoyada por las propias herramientas digitales).

Todo esto, como vemos, tiene como sujeto principal a la mujer, y como objeto principal, datos personales (muchos de ellos especialmente sensibles) que le conciernen. Por ello, la presente contribución se centra en la regulación de la protección de datos a la luz de

\footnotetext{
21 Aludimos a la falta de protección "por diseño" (cogiendo prestada la terminología del RGPD que establece la protección de datos por diseño como una estrategia básica enfocada en la proactividad y prevención en vez de la reactividad y corrección) pero para referirnos a los patrones patriarcales ya citados, a esa cultura tecnológica de la que hablábamos en la Introducción del presente trabajo, que se ve envuelta en la omnipresencia de la tecnociencia en la sociedad, y, subsiguientemente, a esa mentalidad social de titularidad sobre la vida e información de los demás (en particular de las mujeres), y que, precisamente gracias a la tecnología, se extiende sin límite alguno.

${ }^{22}$ Para un análisis y ejemplos concretos de la no neutralidad (y el subsiguiente sesgo de género) de la Red y las herramientas digitales, vid. FERNÁNDEZ, A., "Sesgo de los algoritmos en el Big Data: una perspectiva desde la protección de datos", Politicas públicas en defensa de la inclusión, la diversidad y el género (dir. Gorjón, M.C.), Salamanca, Ediciones Universidad de Salamanca, 2020, pp. 1291-1301.

${ }^{23}$ Vid. BLAZQUEZ, N. y FLORES, J., Ciencia, tecnología y género en Iberoamérica, Ciudad de México, Universidad Autónoma de México-Centro de Investigaciones Interdisciplinarias en Ciencias y Humanidades, 2005, p. 699.

${ }^{24} \mathrm{Vid}$., en líneas similares a mi propuesta, FERREIRO, V., La brecha digital digital, una nueva forma de discriminación hacia las mujeres. La toma de decisión en los usos de internet, Palma, Edicions UIB, 2014.
} 
dos casos particularmente graves de violencia digital contra las mujeres: la violencia sexual digitalizada (grabar y divulgar agresiones y abusos sexuales) y la sextorsión (también conocido en los últimos años como 'porno vengativo'). En el caso de las víctimas de violencia sexual digitalizada, nos encontramos con un doble fundamento jurídico desde la perspectiva de la protección de datos, ya que ni el acto sexual, ni su versión grabada, ni su difusión fueron consentidas. En el caso de las víctimas de pornografía vengativa, si bien los datos personales fueron grabados y compartidos originalmente con su consentimiento, no lo fue su distribución a terceros o su divulgación universal en la Red, a pesar de oponerse a ellos.

\section{APROXIMACIÓN A LA VIOLENCIA DE GÉNERO Y A DOS CASOS DE CIBERVIOLENCIA CONTRA LA MUJER DESDE EL ÁMBITO DE LA PROTECCIÓN DE DATOS}

El marco regulatorio europeo de protección de datos se plantea, y así se establece de manera explícita en el propio Reglamento General de Protección de Datos (en adelante, el RGPD), en clave iusfundamental. Así, se podría argumentar que no sólo confiere un elenco variado de derechos (de acceso, rectificación, cancelación, oposición, supresión, portabilidad, etc.), sino que también da expresión al derecho fundamental a la protección de datos ${ }^{25}$. Esto se ha considerado suficiente para clasificarlo como un sistema de atribución de derechos y como un sistema cuyo diseño e interpretación son consistentes con la concepción subyacente de un derecho fundamental. La naturaleza iusfundamental de este derecho (y, por ende, de su normativa de desarrollo) hace necesario referenciar, aunque sea sucintamente, el contenido esencial del mismo: de una parte, un aspecto (o contenido) negativo en cuanto elemento limitador al tratamiento de datos ${ }^{26} \mathrm{y}$ un aspecto (o contenido) positivo, en cuanto actuación jurídica (o facultad de control), por parte de la persona interesada en el tratamiento, sobre los datos que le conciernen; control que se hace efectivo a través de un abanico de facultades o derechos ${ }^{27}$.

Aunque las mujeres víctimas de violencia de género digital no se mencionan expresamente como grupo vulnerable en el marco europeo de protección de datos, la

\footnotetext{
${ }^{25}$ Como ha dejado sumamente claro el propio Tribunal de Justicia en numerosas sentencias. Vid., por todas, la primera en establecer que la Directiva de protección de datos debe ser interpretada a la luz del art. 8 de la Carta de Derechos Fundamentales de la UE (en adelante, la CDFUE): asuntos acumulados C-465/00, C138/01 y C-139/01, Rechnungshof v. Osterreichischer Rundfunk, STJUE de 20 de mayo de 2003.

${ }^{26}$ Elemento negativo que aparece vertebrado en torno a una serie de principios de los que se desglosan a su vez una serie de derechos y obligaciones; una panoplia de principios de calidad de los datos que regulan y legitiman el tratamiento, de sobra conocidos y ahora resumidos en el RGPD como el principio de exactitud, confidencialidad o seguridad, de finalidad y pertinencia, todo ello basado en el consentimiento (ya previsto en el art. 8.2 CDFUE). Una definición sencilla de cada uno se encuentra disponible en: https://www.aepd.es/es/derechos-y-deberes/cumple-tus-deberes/principios

${ }^{27}$ Sobre la base de un poder de disposición sobre los datos personales se consagraron los clásicos derechos ARCO (acceso, rectificación, cancelación y oposición), ahora ampliados a los derechos "ARSOPOL"(acceso, rectificación, supresión, oposición, portabilidad, oposición a decisiones automatizadas y limitación), como facultades jurídicas de la persona, con su consiguiente obligación jurídica para terceros, de conocer y decidir sobre la propia información personal.
} 
combinación de la perspectiva de riesgo (muy asentada en el nuevo marco europeo de protección de datos), la perspectiva transversal de género (que figura como elemento transversal en la técnica legislativa nacional y europea) y la interpretación sistemática del Derecho de la UE (especialmente, de una lectura conjunta de la Directiva de los derechos de las víctimas de delitos, el RGPD y la Directiva Policial ${ }^{28}$ ) lleva a considerar que el tratamiento de los datos de este colectivo y sus derechos personalísimos gozan de especial protección. Ello se debe no sólo a que la información personal se clasifica, según la normativa de protección de datos, como datos personales especialmente sensibles ('categorías especiales de datos'), sino también a que las mujeres son un colectivo que se ve más envuelto en situaciones de riesgo y especial vulnerabilidad, dentro del formato en línea (y fuera del mismo igualmente facilitado por las NTIC).

En relación a los principios de protección de datos, contenido negativo de este derecho fundamental emergente, cabe destacar lo siguiente. Sobre los principios de limitación y minimización, cabe anunciar que existen autores que ponen en duda su lógica en situaciones de violencia de género: por ejemplo, el principio de minimización "debería entenderse, [...] como principio de maximización de los datos [...] tratar todos y cuantos datos resulten necesarios [...] sin restricciones basadas en un entendimiento positivista absurdo de la normativa, y contrario a las más elementales garantías de la dignidad y de la vida humana" 29 . Resulta totalmente cierto que toda interpretación de la norma jurídica que conduzca a un absurdo debe rechazarse. Ahora bien, nosotros entendemos que los datos que resulten necesarios para el cumplimiento de las funciones de aquellos que tengan que tramitar, y por ende tratar, datos personales no quedarán afectados por este principio, pues recordemos que, al ser la conducta constitutiva de delito, entraría en juego la Directiva Policial como norma aplicable en virtud del principio de especialidad, y esta habla de "no exceso" en lugar de la "no necesidad" del genérico reglamento europeo en la materia. Además, los principios de limitación de la finalidad y minimización de datos requieren también asegurar que el plazo de conservación sea asimismo el mínimo estricto en relación a los fines perseguidos, de tal forma que los datos personales de víctimas de violencia de género solo deberán conservarse por el tiempo que sea necesario, y siempre y cuando siga activa la finalidad para la que se recogieron ${ }^{30}$, estableciéndose unas

\footnotetext{
${ }^{28}$ Directiva 2012/29/UE del Parlamento Europeo y del Consejo de 25 de octubre de 2012 por la que se establecen normas mínimas sobre los derechos, el apoyo y la protección de las víctimas de delitos; el Reglamento 2016/679 del Parlamento Europeo y del Consejo de 27 de abril de 2016 relativo a la protección de las personas físicas en lo que respecta al tratamiento de datos personales y a la libre circulación de estos datos; y Directiva 2016/680 del Parlamento Europeo y del Consejo de 27 de abril de 2016 relativa a la protección de las personas físicas en lo que respecta al tratamiento de datos personales
}

por parte de las autoridades competentes para fines de prevención, investigación, detección o enjuiciamiento de infracciones penales o de ejecución de sanciones penales, y a la libre circulación de dichos datos.

${ }^{29}$ Vid. MARTÍNEZ MARTÍNEZ, R., "Protección de datos y violencia de género. Calidad de los datos, Blog: LOPD y Seguridad, 11-04-2017 (http://lopdyseguridad.es/violencia-de-genero/, consulta: 06/05/2021).

${ }^{30}$ En tal sentido, se debe evitar la cronificación de esta información personal sensible, debiendo los responsables del tratamiento, sobre todo en lo que atañe a organismos públicos y demás entidades que prestan un servicio o ayuda a estas víctimas, a diferenciar entre situaciones que requiere de una intervención 
condiciones y unos plazos para su supresión, en función del potencial ejercicio o defensa de acciones legales presentes o futuras u otros plazos previstos en la legislación nacional que obliguen al mantenimiento de determinados ficheros.

El principio de integridad y confidencialidad exige que el tratamiento se haga con las garantías y medidas técnicas de seguridad ${ }^{31}$, que, en el caso de información tan sensible como posibles agresiones o abusos por razón de género, así como respecto de medidas de protección y seguridad frente a los agresores, implicará indudablemente adecuar medidas físicas y técnicas para preservar la confidencialidad de este tipo de información, en aras de proteger su intimidad y prevenir su identificación o localización; lo que deberá garantizarse tanto en la recogida como en el almacenamiento y cesión de este tipo de información. Sobre esta cuestión, debemos mencionar que el principio de transparencia quedaría limitado en tanto en cuanto el derecho a la información y acceso no puede significar poner en peligro o riesgo a la víctima o su entorno socio-familiar, por lo que quedaría justificada la omisión de cierta información, o incluso la permanencia del anonimato de estas mujeres frente a sus potenciales agresores, actúen o no como investigados o demandados en un procedimiento policial o penal.

Por último, con respecto a la prohibición genérica vertida sobre el tratamiento de datos especialmente sensibles (art. 9 RGPD) como son los concernientes a una víctima de violencia de género, este sí que habla de la posibilidad de su tratamiento "cuando sea estrictamente necesario", cuando se cumplan una serie de excepciones tasadas en el RGPD y la Directiva Policial que legitimarían la recopilación, uso, transmisión o conservación, entre otros, de este tipo de datos de carácter personal. Apreciamos como potenciales supuestos legitimadores del tratamiento los arts. 6(c) del RGPD (cumplimiento de una obligación legal) y los arts. 10(a) y (b) de la Directiva Policial (cuando esté autorizado por la normativa nacional, para el cumplimiento de una obligación legal y cuando sea necesario para proteger los intereses y derechos fundamentales de la víctima u otra persona afectada).

Muchos de los actos que calificamos en su momento como exponentes de la brecha digital por razón de género no son más que ejemplos de un fenómeno más amplio, o si se prefiere, una concreción de la violencia de género, caracterizado por el uso de las NTIC como herramientas para cometer, facilitar o agravar agresiones y abusos de índole sexual, comúnmente llamado "violencia sexual facilitada por la tecnología" (technologyfacilitated sexual violence) $)^{32}$. Algunos autores han criticado el uso de este concepto o de

prolongada en el tiempo y las que son más bien puntuales, pues "una vez agotada la finalidad de la atención, se deberán cancelar los datos de las personas beneficiarias. Para los casos en los que se necesite una atención permanente o extendida en el tiempo hay que valorar si es necesario mantener los datos durante un periodo de tiempo más largo". Vid. INSTITUTO VASCO DE LA MUJER, Guía para el tratamiento de datos...op.cit., p. 61.

${ }^{31}$ Vid. Art. 9.2 (f) del RGPD y art. 4.1(f) de la Directiva Policial.

${ }^{32}$ POWELL, A., y HENRY, N., "Technology-facilitated sexual violence victimization: Results from an online survey of Australian adults", Journal of Interpersonal Violence, Vol. 34, núm. 17, 2016, pp. 3637 3665. 
términos como la 'ciberviolencia' o 'violencia digital' por considerar que el énfasis en la tecnología desvía el foco de atención del debate y apunta al medio utilizado en lugar del problema subyacente de desigualdad de género. Como ya hemos aludido, no es sólo la rapidez y facilidad de acceder a la información confiada (sea de manera consciente, inconsciente o sin consentimiento) en la Red, o la incorporación de cámaras externas de alta definición en los dispositivos móviles, sino también la amplia participación en las redes sociales, así como la falta de sensibilización en materia de género que han contribuido a la comisión de actos violentos, sean de índole psicológica o física, que, en el caso concreto de las mujeres, han tendido a reforzar estereotipos y dobles estándares ${ }^{33}$ y a materializarse a través de actos de índole sexual.

Este asalto a la dignidad e integridad se intensifica, produciendo un trastorno de estrés postraumático (en adelante, TEPT) digital con graves repercusiones psicológicas y haciendo el camino a la recuperación un imposible para aquellas que las sufren: a saber, las víctimas de la pornografía vengativa y víctimas de violencia sexual cuyas agresiones o abuso ha sido grabado y distribuido por la Red. Existe, desde luego, una tendencia preocupante en la que las NTIC se utilizan como herramienta para el abuso, intimidación y control de las mujeres ${ }^{34}$. En este tipo de fenómeno, el trauma es doble y las posibilidades de re-victimización aumentan considerablemente. Por un lado, la mujer no sólo se encuentra envuelta en un acto sexual no consentido, sino que este, seguidamente, se difunde, de nuevo sin su consentimiento, por la mensajería instantánea de sus agresores, $\mathrm{u}$ otras aplicaciones digitales o la propia Red, perdiendo toda su intimidad y toda capacidad de control sobre la información que le concierne vertida en los recovecos digitales (desde su nombre hasta su imagen). De hecho, existen individuos que revelan información personal de estas víctimas y difunden intencionadamente contenidos comprometidos (teniendo ahora que añadir también a la realidad actual el fenómeno de las "manadas virtuales").

En el caso de las víctimas de pornografía vengativa, encontramos una situación similar. Si bien la información personal de contenido íntimo o sexual ${ }^{35}$ suele cederse, en su origen, con el consentimiento de la víctima, es la difusión no consentida a terceros la que resulta problemática; suele enmarcarse en el contexto de la ruptura de una relación íntima, y así, se concibe como práctica vengativa (de ahí su nombre estadounidense, revenge porn) o práctica de extorsión, demandando cualquier tipo de acto u omisión para no ponerlas a

\footnotetext{
${ }^{33}$ Vid., por todos, RINGROSE J., GILL R., LIVINGSTONE S., y HARVEY, L., A qualitative study of children, young people and 'sexting': report prepared for the NSPCC, 2012, pp. 7 y 45-47.

${ }^{34}$ Vid., LARRÁYOZ SOLA, I., "La amenaza de difundir un vídeo es intimidación típica de la agresión sexual: STS 432/2018, de 28 septiembre 2018”, Revista Aranzadi Doctrinal, núm. 11, 2018.

${ }^{35}$ Suelen ser fotografías enviadas, mediante la práctica del sexting, y varían desde contenidos sugestivos o provocativos hasta desnudez total o parcial, seguida de otros datos personales además de la imagen, tales como el nombre, el correo o el usuario en las redes sociales o su teléfono móvil. Vid. WALKER, K., y SLEATH, E., "A systematic review of the current knowledge regarding revenge pornography and nonconsensual sharing of sexually explicit media", Aggression and Violent Behavior, Vol. 36, 2017, p. 9.
} 
disposición del público, aunque no siempre es el caso ${ }^{36}$. En este sentido, algunos consideran más apropiado hablar de "abuso" o "explotación sexual basada en imágenes" "37. Las últimas cifras demuestran que, por ejemplo en Facebook, se denuncian 54.000 casos al mes relativos a este tipo de conducta ${ }^{38}$. Quizás lo que hace esta conducta tan horrenda no es solo "la invasión terrible de la intimidad sexual y el espacio personal" sino también que "en la mayoría de los casos se ha llevado a cabo por quien en su día fue un ser querido y una persona de confianza" 39 . Aquí, a pesar de no haber dos actos traumáticos seguidos, pues, en principio, la fotografía o video provocativa $o$ representando un acto de índole sexual en su origen se realizó y se envió mediando consentimiento por parte de la mujer, la divulgación posterior (y su imparable viralización) es igualmente una injerencia ilegítima en sus derechos personalísimos, en especial el derecho a la intimidad y a la protección de datos, y se asocia también a niveles altos de síntomas psiquiátricos tales como TEPT, depresión, ansiedad, neurosis, trastornos del sueño, bajos niveles de autoestima, desconfianza en las personas y en la tecnología, sentimientos de vergüenza y autoculpa, etc ${ }^{40}$.

Además de los daños psicológicos asociados a este fenómeno, las víctimas de este tipo de ciberviolencia sexual sufren diversos daños irreparables que afectan a su vida personal $\mathrm{y}$, sobre todo, profesional. Relaciones y familias quedan destrozadas y los medios de subsistencia en ocasiones se ven comprometidos ${ }^{41}$. En efecto, como hemos dicho en varias ocasiones, la universalización y perennidad de la Red no solo dificulta sino que, en determinadas circunstancias, imposibilita la capacidad de desprenderse de eventos pasados, inherentemente discriminatorios y especialmente traumáticos. Con respecto a otras garantías que ofrece la normativa europea en materia de protección de datos, debemos hablar del derecho al olvido como facultad de actuación jurídica y como garantía efectiva para la protección de este colectivo ante estas situaciones de especial riesgo de vulnerabilidad socio-digital.

\footnotetext{
${ }^{36}$ Aunque, como acertadamente apuntan algunos autores, el porno vengativo no necesariamente se hace como práctica vengativa ni por parte de una persona conocida. Vid. INSTITUTO EUROPEO DE IGUALDAD DE GÉNERO, Cyberviolence against women...op.cit., p. 2.

${ }^{37}$ POWELL, A., y HENRY, N., Sexual Violence in a Digital Age, Palgrave...op.cit., p. 118; y MCGLYNN, C., RACKLEY, E. y HOUGHTON, R., "Beyond 'Revenge Porn': The Continuum of Image-Based Sexual Abuse", Feminist Legal Studies, Vol. 25, 2017, pp. 25-46.

38 OTTO, C., "El infierno de la 'sextorsión': Mi exnovio llenó internet de fotos mías desnuda", $E l$ Confidencial, 03-07-2017 (https://www.elconfidencial.com/tecnologia/2017-07-03/sextorsion-pornovengativo-porn-revenge-delitos_1406269/, consulta: 06/05/2021).

${ }^{39}$ BATES, S., "Revenge porn and mental health: A qualitative analysis of the mental health effects of revenge porn on female survivors", Feminist Criminology, Vol. 12, 2016, p. 38.

${ }^{40}$ STAUDE-MÜLLER, F., HANSEN, B. y VOSS, M., "How stressful is online victimization? Effects of victim's personality and properties of the incident", European Journal of Developmental Psychology, Vol. 9, núm. 2, 2012, p. 263.

${ }^{41}$ No son pocos los casos en los que se ha recurrido a despidos disciplinarios por culpa de fotos que se han publicado o distribuido por las redes sociales sin el consentimiento de la afectada.
} 


\section{EL 'DERECHO AL OLVIDO' COMO GARANTÍA JURÍDICA Y DIGITAL: PROPUESTAS DE FERENDA Y LEGE DATA PARA ASEGURAR LA DIGNIDAD DE LAS MUJERES VÍCTIMAS DE CIBERVIOLENCIA SEXUALIZADA}

El acceso a cierta información personal, sobre todo aquella prevista para ser visualizada en el exclusivo contexto de una relación íntima, que la vincula, en el actual paradigma tecno-digital, eternamente con un nombre, un usuario y una serie de enlaces, perpetúa el trauma de estas mujeres, prolonga el duelo, cercena su libre desarrollo en sociedad y hace de la recuperación a menudo un imposible. Por ello, es necesario un mecanismo efectivo que permita a estas mujeres no quedar atrapadas en un túnel Funesiano en el que el recuerdo del evento traumático se convierta en "fantasma obsesivo" o "arma arrojadiza vengativa" 42 . Este mecanismo no es otro que el derecho al olvido ${ }^{43}$, como garantía de una 'evitación' (digital).

Así lo he estudiado a fondo, proponiendo dos derechos (o subcategorías de un mismo derecho) diferenciados. De hecho, la propuesta de dos derechos al olvido no es novedosa, pues, por un lado, el propio RGPD daba pie a confusión (o, a entendimientos alternativos) al diferenciar, a lo largo de su articulado, el derecho de supresión del derecho al olvido, aunque ambos quedasen oficialmente regulados como una figura en su art. 17. De una minuciosa lectura de las disposiciones de la norma observamos que, en la parte expositiva del RGPD, encontramos alusiones a un derecho al olvido como ampliación del derecho de supresión, hablando de la supresión de enlace, copias o réplicas en el entorno en línea(Considerando 66 RGPD), alusiones a dos derechos bien diferenciados cuando se habla del ejercicio de las facultades del interesado o, concretamente "del derecho de supresión, [del derecho derecho] al olvido” (Considerando 156 RGPD), e incluso distinguiendo, dentro su propio listado de definiciones, entre supresión y destrucción (art. 4.2 RGPD). Por otro lado, si bien la parte expositiva de la Ley Orgánica de Protección de Datos y Garantías de los Derechos Digitales (en adelante, la LOPDGDD) se centra en un derecho de supresión, su parte dispositiva también ofrece distintos derechos al olvido: para el derecho de supresión (art. 15 LOPDGDD) se remite al artículo correspondiente de la norma europea, titulado "derecho de supresión ('derecho al olvido')" y establece, como ya hemos visto, un derecho al olvido en dos entornos digitales concretos, en búsquedas de Internet y en servicios de redes sociales y servicios equivalentes (arts. 93 y 94 LOPDGDD).

De tal modo, consideramos necesario establecer una clara diferenciación entre un derecho de supresión de información personal almacenada (sea esta digital o no), y un derecho a una suerte de restauración digital que libera a la persona de las cadenas de un pasado que

\footnotetext{
42 ECHEBURÚA ODRIOZOLA, E., y CRUZ-SÁEZ, M.S., "De ser víctimas a dejar de serlo: un largo proceso", Revista de Victimología, núm. 1, 2015, p. 93.

43 Vid. MARTÍNEZ LÓPEZ-SÁEZ, M., La garantía del derecho al olvido: protección de datos, retos futuros y propuestas de regulación de situaciones de vulnerabilidad en la Unión Europea, Tesis Doctoral, Valencia, Universidad de Valencia, 2020.
} 
no se corresponde con la realidad, pero que consta, de manera instantánea, universal y descontextualizada, como la única verdad presente para cualquiera que la busca en Internet (lo que hemos decidido llamar "derecho al libre desarrollo de la identidad digital" o a la "re-contextualización digital"). En cualquier caso, ambos pretenden ofrecer, como elocuentemente propuso MAYER-SCHÖNBERGER, un "principio de ecología"44 en el ámbito de la mutada informática (y nosotros añadiríamos en una sociedad todavía plagada de estructuras culturales y relaciones sociales ancladas en la desigualdad).

Así, el derecho al olvido jugaría un papel importante no sólo en el restablecimiento del control de este colectivo sobre su propia información personal (de carácter íntimo, además) y como mecanismo de empoderamiento socio-digital, sino también como herramienta para el alivio del trauma constante que supone tener un rastro digital eterno e incesante de la Red y para poder llegar más fácilmente a una recuperación psicoemocional, sin tener que tomar medidas tan drásticas como cambiarse su nombre, usuario o desaparecer de las redes sociales, servicios digitales o incluso cambiar de ciudad.

Recordemos que, a nivel europeo, contamos con dos instrumentos normativos en materia de protección de datos, que reconocen, en su concreto ámbito de su aplicación, un derecho al olvido. En materia de cooperación policial y judicial en el ámbito penal, es decir, cuando la víctima denuncia, contamos con la Directiva Policial, que no sólo hace referencia a la protección de colectivos en situación de especial vulnerabilidad frente a riesgos y daños psicológicos del proceso ${ }^{45}$ (entre los que indudablemente incluimos a las víctimas de ciberviolencia sexual), sino que también presenta, como principal objetivo, encontrar el equilibrio entre la libre circulación de datos de carácter personal y el derecho fundamental a la protección de datos. Al igual que con las víctimas de violencia de género, en el caso de víctimas de violencia de género sexual y digitalizada, la norma obliga a garantizar un nivel de seguridad y confidencialidad de los datos que se traten, e incluye el derecho de supresión cuando ya no sean necesarios para los fines para los que fueron recabados en su origen, o, en su defecto, el derecho a la limitación del tratamiento (arts. 16.3 in fine y 16.4 de la Directiva Policial).

En lo que se refiere específicamente al derecho al olvido, observamos que la norma lo contempla en su faceta de derecho de supresión, para el que encontramos un par de supuestos habilitadores: cuando la supresión sea una obligación legal y cuando el tratamiento se haya realizado vulnerando cualquiera de los principios de protección de datos, siendo, por ende, ilícito (art. 16.2 de la Directiva Policial). No obstante, es de sobra conocido que, ante delitos como son los adscritos a este tipo de ciberviolencia, las autoridades competentes, fuera de lo que son medidas de investigación tecnológicas que ellos mismos como responsables del tratamiento garantizan que se realicen acorde con la

\footnotetext{
${ }^{44}$ Vid. MAYER-SCHÖNBERGER, V., Delete. The Virtue of Forgetting in the Digital Age, Princeton University Press, New Jersey, Princeton University Press, 2009, pp. 157-164.

${ }^{45} \mathrm{Vid}$. Considerandos 39, 50 y 75 del RGPD.
} 
normativa de protección de datos ${ }^{46}$, aseguran también, como medida cautelar, el bloqueo o la prohibición de seguir difundiendo el contenido dañoso. Tal y como nos recuerdan algunos autores, el objetivo es evitar que se siga produciendo un daño a la víctima, agravando el ya estudiado TEPT digital, siendo ambas consecuencias lesivas y continuadas del delito ${ }^{47}$.

No obstante, con independencia de que la víctima de este tipo de violencia denuncie su agresión u abuso sexual o tecnológico, y ello devenga, o no, en un proceso judicial, el responsable del tratamiento, sea una red social o un motor de búsqueda, también estará obligado a la supresión o a la desindexación, si así lo solicita la víctima, cuando se dé uno de los supuestos que contempla el art. 17 RGPD, el segundo instrumento normativo europeo relevante en materia de protección de datos.

Así, fuera del contexto policial y judicial, debemos, en primer lugar, hablar de la falta de consentimiento como uno de los títulos habilitantes para ejercer el derecho al olvido, en cualquiera de sus facetas, pues el principio del consentimiento determina la licitud del tratamiento ${ }^{48}$, por lo que la vulneración del mismo resultará en un tratamiento ilícito. El derecho al olvido, directamente ligado con el consentimiento, actúa como mecanismo de empoderamiento y autodeterminación en el control de los datos de carácter personal, y como garantía para eliminar o impedir el acceso y utilización sucesiva de datos personales, precisamente cuando su tratamiento deja de estar (o, como en este caso, nunca estuvo) autorizado por el interesado (art. 17.2 RGPD). Las víctimas de ciberviolencia sexual no tienen por qué soportar la omnipresencia de sus datos personales (sea su imagen, su nombre, o cualesquiera otra información identificativa) en la Red, sobre todo cuando no existe interés alguno, o suficiente, que justifique su permanencia.

En efecto, la falta de condición de personaje público de la víctima y la falta de necesidad de revelar su identidad o contenidos tan sensibles como el video de su agresión o su nombre completo, DNI, o los usuarios de las cuentas digitales que poseía, a pesar de quizás ser un hecho noticiable o de interés periodístico, hace que el tratamiento de estos datos personales se considere desproporcionado y excesivo. Este fue el caso, en España, de la filtración de los datos personales de la víctima de la Manada de Pamplona, y Google, como motor de búsqueda y responsable del tratamiento, aplicó el derecho al olvido digital, eliminando los enlaces y su localización en su página de resultados tras una búsqueda nominativa ${ }^{49}$. Según otras fuentes, los demás buscadores populares en España tardaron

\footnotetext{
${ }^{46}$ Ello incluye, pero no se limita a, interceptación de las comunicaciones telefónicas y telemáticas, captación y grabación de comunicaciones, captación de imagen seguimiento y localización, registro de dispositivos o equipos...Vid., en tal sentido, PALOP BENLLOCH, M.: Protección jurídica de menores víctimas de violencia...op.cit. pp. 129-166.

${ }^{47}$ MORENO CATENA, V., Derecho procesal penal, Valencia, Tirant lo Blanch, 2015, pp. 309-311.

${ }^{48}$ Se consagra como premisa para la licitud y legitimación del tratamiento según lo establecido en el art. 6.1(a) RGPD.

49 Vid., por todas las noticias, CASTRO, N, "Google borra los datos filtrados de la víctima de "La Manada", El Plural, 04-05-2018 (https://www.elplural.com/sociedad/google-borra-los-datos-filtrados-de-la-victimade-la-manada_127319102, consulta: 06/05/2021)
} 
más en hacer desaparecer su rastro, lo que supuso que, según la herramienta 'Google Trends' (que cuantifica el interés que registra la búsqueda de un término concreto), en cuestión de 24 horas, la popularidad máxima de su nombre fuera del cien por cien ${ }^{50}$. Ello no sólo produjo una revictimización tan grave que la obligó a someterse a diferentes tratamientos psicológicos, sino también al abandono de sus estudios e incluso a su huida al extranjero. Por ello, compartimos las reflexiones DE VERDA Y BEAMONTE en lo que se refiere a la protección absoluta del anonimato de víctimas de delitos gravemente atentatorios contra la dignidad (en los que incluye los delitos sexuales y la violencia contra la mujer) y en sus reflexiones acerca de la proyección social no habitual (sino que es forzada por su condición de víctima en un proceso judicial mediático) en lo que ataña a su identificación; se trata de una identificación que califica de intolerable "ya que la revelación de su identidad no era necesaria para informar a la sociedad del suceso"

De hecho, damos un paso más. Por un lado, proponemos el derecho al olvido como mecanismo para prevenir la victimización secundaria y evitar intromisiones indebidas a su intimidad, pues funciona como mecanismo de eliminación y de disociación de los recuerdos del evento traumático (o en sí, del propio evento), y, por ende, como herramienta para el mantenimiento del equilibrio emocional. Por otro, consideramos que la excepción periodística que contempla la regulación del derecho al olvido en el art. 17.3(a) RGPD no es aplicable en estos supuestos puesto que, la necesidad de informar sobre la identidad de la víctima que sufre un suceso violento de trascendencia social no supera el examen de proporcionalidad, y, en muchas ocasiones, dicha revelación y divulgación de información personal nutre más la satisfacción de la curiosidad mórbida y produce juicios mediáticos y sociales paralelos ${ }^{52}$. Así, al igual que se debe mirar la naturaleza del delito, también debe mirarse la situación especial de la víctima que sufre sus consecuencias (la "situación particular" de la persona afectada ${ }^{53}$ ), y, ante la duda, en la era digital actual, ofrecerle las mayores garantías a su intimidad y su derecho fundamental a la protección de datos, que no tienen otro objetivo que garantizar su seguridad, integridad y dignidad.

En el caso de las víctimas de violencia sexual digitalizada, nos encontramos con doble fundamento, pues ni el acto sexual fue consentido ni el material audiovisual fue grabado

\footnotetext{
${ }^{50}$ EL CONFIDENCIAL, "Google borra las pistas que permiten identificar a la víctima de 'La Manada", El Confidencial, 04-05-2018 (https://www.elconfidencialdigital.com/articulo/vivir/Google-permitenidentificar-victima-Manada/20180503174719089349.html , consulta: 06/05/2021)

${ }^{51}$ DE VERDA BEAMONTE, J.R., "Libertad de información y derecho a la intimidad de las víctimas. Comentario de la STS (Pleno) núm. 91/2017, de 15 de febrero", Actualidad Jurídica Iberoamericana, núm. 6 (febrero), 2017, pp. 282-284.

${ }^{52}$ Ese "afán voraz del público por acceder a cualquier detalle" y a los "juicios paralelos" o "transformación del proceso mismo en una especie de espectáculo informativo". Vid. SERRA CRISTOBAL, R., "Los derechos de la víctima en el proceso penal vs. medios de comunicación. Especial referencia a las víctimas de violencia por motivos de género", Revista española de derecho constitucional, Vol. 35, núm. 103, 2015, pp. 199-230.

${ }^{53}$ Por utilizar la terminología pro personae por la que ha optado en los últimos años el TJUE al interpretar la antigua Directiva de Protección de Datos y el actual RGPD.
} 
con su consentimiento (y mucho menos lo fue su difusión). En el caso de las víctimas de pornografía vengativa, si bien el material audiovisual fue grabado y compartido mediando consentimiento, su distribución a terceros o su publicación en abierto en la Red no lo fue. A tal efecto, las redes sociales contemplan la supresión de contenidos de esta índole en sus directrices internas y normas de la red social. En lo que nos interesa, Facebook ${ }^{54}$, por ejemplo, tiene como política interna (así al menos consta en su 'libro de normas' sobre las publicaciones que permite en su red social) eliminar: (i) contenidos que van en contra de la seguridad personal, es decir, que produzcan riesgo de daños físicos, emocionales o financieros (Safety), incluyendo imágenes de violencia sexual en adultos, explotación sexual de menores (tal y como imágenes de menores desnudas o semi-desnudas) o cualquier otro tipo de imagen no consentida por la persona afectada (Privacy Violations and Image Privacy Rights); o (ii) contenidos considerados ofensivos (Objectionable content) tales como aquellos que glorifican la violencia, desnudez y actividad sexual en adultos y contenidos que consideren crueles e insensibles, incluidos aquellos dirigidos a víctimas de daños físicos o emocionales ${ }^{55}$.

Sin embargo, si bien es cierto que, en ocasiones, esto ha supuesto la eliminación de contenidos inofensivos o necesarios para el debate público, lo que ciertas voces críticas han calificado como censura ${ }^{56}$, consideramos esta práctica como protectora de los derechos fundamentales de las víctimas de violencia de género digital y articuladora del principio favor libertatis (o si se prefiere, pro personae), pues, como hemos visto, la eliminación de contenidos publicados en servicios digitales es reversible, mientras que la reputación y salud mental de aquellas que sufren la publicación y distribución de contenido tan nocivo no lo es; estas personas (mujeres en su mayoría) no pueden permitirse el error (ni deben asumir cadenas perpetuas) de que sus datos personales e imágenes tan comprometidas, fueran consentidas o no en su origen, queden universal e ilimitadamente accesibles.

Así también parecen haberlo entendido las agencias y autoridades independientes para estos fenómenos concretos de ciberviolencia sexual. De hecho, en numerosas ocasiones han tenido que intervenir y colaborar con las autoridades judiciales y policiales para evitar mayor disponibilidad y accesibilidad a este tipo de contenidos. Por citar el caso español, la Agencia Española de Protección de Datos (en adelante, la AEPD) el año pasado inauguró un espacio web de ayuda a la protección de la privacidad de las víctimas de ciberviolencia de género en el contexto de acoso digital, sextorsión o pornografía vengativa. Ello incluye no sólo información práctica para proteger su privacidad, qué

\footnotetext{
${ }^{54}$ Facebook se basa en denuncias de este tipo de contenido (flagged content) y simultáneamente hace uso de sistemas de Inteligencia Artificial y equipos de revisores para una identificación y ejecución más eficaz de sus estándares comunitarios.

55 FACEBOOK, Community Standards, Facebook, 2018. Disponible en: https://www.facebook.com/communitystandards/

${ }^{56}$ HERNÁNDEZ BORBOLLA, M., "Censura y control de internet: ¿qué poder y alcance tienen Facebook, Google y Twitter?", RT, 17-09-2019. Disponible en: https:/actualidad.rt.com/actualidad/327362-censuracontrol-internet-poder-redes-sociales
} 
servicios están a su alcance y cómo proceder, sino también, y en lo que concierne al tema objeto de estudio, a través de un canal específico, facilitar y expeditar los procedimientos de retirada urgente de contenidos sexuales o violentos, difundidos ilícitamente tras su comunicación ${ }^{57}$. Así, observamos que el derecho al olvido como derecho de supresión (o retirada de contenidos nocivos) y el derecho al olvido digital como desvinculación de datos personales con esos contenidos nocivos supone devolverle a la víctima de ciberviolencia sexual un poder de control y un poco de dignidad, saqueada en el mundo virtual por razones perversas e injustificables por su agresor ${ }^{58}$. En efecto, la normativa de protección de datos contribuye a proteger la seguridad de este colectivo a través de numerosos mecanismos que, tanto a priori, como a posteriori (como es el caso del derecho al olvido), y junto con otras medidas preventivas necesarias fuera del ámbito de la protección de datos, evitan o, al menos, reducen los daños físicos, psíquicos, familiares, sociales, laborales o económicos experimentados, tanto por las conductas reprochables de los perpetradores como por la re-victimización derivada de un inadecuado tratamiento de su información personal, incluso por aquellos designados a ayudar y protegerlas.

Una de estas garantías, en tanto facultad de actuación jurídica, y parte del contenido positivo del derecho fundamental ${ }^{59}$ a la protección de datos, es el derecho al olvido, ya sea entendido como derecho al borrado de datos (o retirada de los contenidos lesivos) o como derecho a la descontextualización o "re-contextualización"60 (desvinculando los enlaces con contenidos lesivos basados en datos personales), supone devolver a la víctima de la ciberviolencia sexual un poder de control y algo de dignidad, aquella que fue saqueada en el mundo virtual por razones perversas e injustificables por sus agresores $u$ otros interesados en opinar sobre tal afrenta a su dignidad personal.

\section{CONCLUSIÓN}

Si bien somos seres de carne y hueso, parte de nuestra vida está en la Red, troceada, desordenada y desperdigada en bits y bytes, pero fácilmente recuperable y presentada en

\footnotetext{
${ }^{57}$ Sobre este servicio, la propia AEPD destaca que "Nuestra imagen, [...] fotos y videos [...] es un dato personal y para su difusión en los diferentes servicios de internet debe existir nuestro consentimiento u otra base legal. La Agencia [...] ]encargada de tutelar el derecho de supresión en caso de que el responsable de la publicación de contenidos en redes sociales y otros sitios [...] no responda a la solicitud de supresión [...]En situaciones excepcionalmente delicadas, cuando las imágenes tengan contenido sexual o muestren actos de agresión [...]poniendo en alto riesgo los derechos y libertades [...] especialmente víctimas de violencia de género [...] prestadores de servicios online pueden no resultar suficientemente eficaces y rápidos para evitar la difusión continuada de las imágenes [...] Las reclamaciones recibidas por este canal serán analizadas de forma prioritaria [...] si fuera preciso, la adopción de medidas urgentes que limiten la continuidad del tratamiento de los datos personales".

${ }^{58}$ Que, recordemos además son también responsables del tratamiento, según la jurisprudencia del Asunto C-101/01, Lindqvist, STJUE de 6 de noviembre de 2003, quedando expuestos a las sanciones pertinentes por el incumplimiento de la normativa de protección de datos, además de las correspondientes a las demás infracciones penales.

${ }^{59}$ Así sea reconocido no sólo a nivel europeo (de manera directa del art. 8 CDFUE y, de manera indirecta, a través de la jurisprudencia del TEDH del art. $8 \mathrm{CEDH}$ ), sino también nacional (art. $18.4 \mathrm{CE}$ y su interpretación por parte del TC desde las sentencias 290/2000 y 292/2000 de 30 de noviembre.
}

${ }^{60}$ Así lo he propuesto en MARTÍNEZ LÓPEZ-SÁEZ, M., La garantía del derecho al olvido...op.cit. 
un formato estructurado (si bien fuera de su contexto) en cuestión de un solo clic. Una vida libre de agresiones e intimidaciones (presenciales o a distancia) no sólo es un derecho fundamental de toda persona, sino que en el caso de las mujeres afectadas por la violencia (incluida la digital), esta máxima axiológica requiere de especiales garantías que aseguren su igualdad, libertad, y, en última instancia, su dignidad. Ante las nuevas realidades sociodigitales, el Derecho se ha ido adaptando y el marco europeo de protección de datos se ha ampliado para ofrecer nuevos mecanismos de protección.

En el caso específico del derecho al olvido, garantía específica (facultad de actuación jurídica parte del contenido positivo) del derecho fundamental a la protección de datos, se pretende restaurar la capacidad de control de la propia información personal, frente a situaciones digitales y relaciones sociales que, por su propia naturaleza, nacen desequilibradas, convirtiéndose no sólo en una garantía de autodeterminación informativa, y de empoderamiento, sino también en una garantía de recuperación de algunos de los males más atroces de la era digital actual. En el caso específico de la mujer, como víctima de ciberviolencia (general y desgraciadamente sexualizada), esta requiere de salvaguardas específicas.

Así las cosas, las mujeres víctimas de violencia de género constituyen un colectivo en situación de especial vulnerabilidad en el entorno virtual, y, por ende, son beneficiarias necesarias de la protección que supone la garantía del derecho al olvido, como arma digital de empoderamiento y resiliencia, para ubicarlas en condiciones de igualdad efectiva, con el fin de evitar una eventual re-victimización. Una de estas salvaguardas específicas estudiadas ha sido el derecho al olvido, bien entendido como el borrado de datos personales o como la desvinculación de enlaces o contenidos indexados a través de datos personales. Este derecho protege una amalgama muy variada de derechos fundamentales $\mathrm{y}$, en lo que a las víctimas de la violencia de género digital se refiere, además responde al principio pro personae.

Por ello, esta contribución se ha centrado en examinar la configuración del derecho al olvido y la re-configuración de otros aspectos de la normativa actual de protección de datos ante los desafíos de dos casos concretos de violencia digital contra la mujer, con el fin de revertir situaciones impuestas de vulnerabilidad e indefensión derivada de la especificidad de la realidad socio-digital y con el fin de asegurar un empoderamiento femenino y una igualdad efectiva, contribuyendo a hacer del mundo virtual (igual de real y relevante que el de carne y hueso) uno más igualitario, justo y más digno.

\section{BIBLIOGRAFÍA}

AGENCIA DE LOS DERECHOS FUNDAMENTALES DE LA UE, Violencia de género contra las mujeres: una encuesta a escala de la UE. Resumen de las conclusiones, Oficina de Publicaciones de la Unión Europea, Luxemburgo, 2014, https://fra.europa.eu/es/publication/2020/violencia-de-genero-contra-lasmujeres-una-encuesta-escala-de-la-ue-resumen-de-las 
BATES, S., "Revenge porn and mental health: A qualitative analysis of the mental health effects of revenge porn on female survivors", Feminist Criminology, Vol. 12, 2016, pp. 22-42.

BLAZQUEZ GRAF, N. y FLORES, J., Ciencia, tecnología y género en Iberoamérica, Ciudad de México, Universidad Autónoma de México-Centro de Investigaciones Interdisciplinarias en Ciencias y Humanidades, 2005.

CASTRO, N, "Google borra los datos filtrados de la víctima de 'La Manada", El Plural, 04-05-2018, https://www.elplural.com/sociedad/google-borra-los-datosfiltrados-de-la-victima-de-la-manada_127319102

DE VERDA BEAMONTE, J.R., "Libertad de información y derecho a la intimidad de las víctimas. Comentario de la STS (Pleno) núm. 91/2017, de 15 de febrero", Actualidad Jurídica Iberoamericana, núm. 6 (febrero), 2017, pp. 278-291.

ECHEBURÚA ODRIOZOLA, E., y CRUZ-SÁEZ, M.S., "De ser víctimas a dejar de serlo: un largo proceso", Revista de Victimología, núm. 1, 2015, pp. 83-96.

EL CONFIDENCIAL, "Google borra las pistas que permiten identificar a la víctima de 'La Manada'", El Confidencial, 04-05-2018, https://www.elconfidencialdigital.com/articulo/vivir/Google-permitenidentificar-victima-Manada/20180503174719089349.html

ESTEBÁNEZ, I. y VÁZQUEZ, N., La desigualdad de género y el sexismo en las redes sociales. Una aproximación cualitativa al uso que hacen de las redes sociales las $y$ los jóvenes de la CAPV, Vitoria-Gasteiz, Servicio Central de Publicaciones del Gobierno Vasco, 2013,

FERNÁNDEZ, A., "Sesgo de los algoritmos en el Big Data: una perspectiva desde la protección de datos", Políticas públicas en defensa de la inclusión, la diversidad y el género (dir. Gorjón, M.C.), Salamanca, Ediciones Universidad de Salamanca, 2020, pp. 1291-1301.

FERREIRO, V., La brecha digital digital, una nueva forma de discriminación hacia las mujeres. La toma de decisión en los usos de internet, Palma, Edicions UIB, 2014.

FERREIRO, V., VILÀ, R., y PRADO, N., "Experiencias sexistas en las redes sociales. Perpetuando la violencia de género", Psicología y Educación: Presente y Futuro (coord. Castejón, J.L.), Alicante, ACIPE, 2016, pp. 582-590.

FLORES FERNÁNDEZ, J., "Privacidad, factor de riesgo y protección en la violencia digital contra las mujeres", Ciberacoso y violencia de género en redes sociales: Análisis y herramientas de prevención (coord. Verdejo, M.A.), Sevilla, Universidad Internacional de Andalucía, 2015, pp. 313-321. 
HERNÁNDEZ BORBOLLA, M., "Censura y control de internet: ¿qué poder y alcance tienen Facebook, Google y Twitter?”, RT, 17-09-2019, https://actualidad.rt.com/actualidad/327362-censura-control-internet-poderredes-sociales

HARRIS, B., "Spacelessness, Spatiality and Intimate Partner Violence: Technologyfacilitated Abuse, Stalking and Justice”, en AA. VV. Intimate Partner Violence, Risk and Security: Securing Women's Lives in a Global World, Nueva York y Oxon, Routledge, 2018, pp. 52-70.

INSTITUTO EUROPEO DE IGUALDAD DE GÉNERO, Cyber violence against women and girls, Oficina de Publicaciones de la Unión Europea, Luxemburgo, 2017, https://eige.europa.eu/publications/cyber-violence-against-women-and-girls

LARRÁYOZ SOLA, I., "La amenaza de difundir un vídeo es intimidación típica de la agresión sexual: STS 432/2018, de 28 septiembre 2018", Revista Aranzadi Doctrinal, núm. 11, 2018.

MARTÍNEZ LÓPEZ-SÁEZ, M., La garantía del derecho al olvido: protección de datos, retos futuros y propuestas de regulación de situaciones de vulnerabilidad en la Unión Europea, Tesis Doctoral, Valencia, Universidad de Valencia, 2020.

MARTÍNEZ MARTÍNEZ, R., "Protección de datos y violencia de género. Calidad de los datos, Blog: LOPD y Seguridad, 11-04-2017, http://lopdyseguridad.es/violenciade-genero

MASFERRER DOMINGO, A., "Vulnerability and Dignity in the Age of Rights", en AA. VV., Human Dignity of the Vulnerable in the Age of Rights. Interdisciplinary Perspectives, Basel, Springer, 2016, pp. 1-29.

MAYER-SCHÖNBERGER, V., Delete. The Virtue of Forgetting in the Digital Age, Princeton University Press, New Jersey, Princeton University Press, 2009.

MCGLYNN, C., RACKLEY, E. y HOUGHTON, R., "Beyond 'Revenge Porn': The Continuum of Image-Based Sexual Abuse", Feminist Legal Studies, Vol. 25, 2017, pp. 25-46.

MONTIEL JUAN, I., "Cibercriminalidad social juvenil: la cifra negra", Revista de los Estudios de Derecho y Ciencia Política (IDP), núm. 22, 2016, pp. 119-131.

MORENO CATENA, V., Derecho procesal penal, Valencia, Tirant lo Blanch, 2015.

OCDE, Report: Bridging The Digital Gender Divide. Include. Upskill. Innovate, OCDE, 2018, https://www.oecd.org/digital/bridging-the-digital-gender-divide.pdf 
OMS, Resumen de orientación Estimaciones mundiales y regionales de la violencia contra la mujer: prevalencia y efectos de la violencia conyugal y de la violencia sexual no conyugal en la salud, OMS, Ginebra, 2013.

OTTO, C., "El infierno de la 'sextorsión': Mi exnovio llenó internet de fotos mías desnuda", El Confidencial, 03-07-2017, https://www.elconfidencial.com/tecnologia/2017-07-03/sextorsion-pornovengativo-porn-revenge-delitos_1406269

PALOP BENLlOCH, M., Protección jurídica de menores víctimas de violencia de género a través de Internet. Vulnerabilidad de la menor en sus relaciones de pareja, ciberacoso y derecho al olvido, Cizur Menor, Thomson Reuters-Aranzadi, 2019.

PERONI, L. y TIMMER, A., "Vulnerable groups: The promise of an emerging concept in European Human Rights Convention Law", International Journal of Constitutional Law, Vol. 11, núm. 4, 2013, pp. 1056-1085.

POWELL, A., y HENRY, N., Sexual Violence in a Digital Age, Melbourne, Palgrave MacMillan, 2017.

POWELL, A., y HENRY, N., "Technology-facilitated sexual violence victimization: Results from an online survey of Australian adults", Journal of Interpersonal Violence, Vol. 34, núm. 17, 2016, pp. 3637-3665.

QUESADA AGUAYO, M.S., "La violencia de género y el ciberacoso en las redes sociales: análisis y herramientas de detección”, Ciberacoso y violencia de género en redes sociales: Análisis y herramientas de prevención (coord. Verdejo, M.A.), Sevilla, Universidad Internacional de Andalucía, 2015, pp. 111-226.

QUESADA AGUAYO, M.S., "Género y discriminaciones asociadas al hecho de ser mujer", Manual Agentes de Igualdad (coord. Román Onsalo, M.), Sevilla, Diputación de Sevilla, 2009, pp. 30-42.

RIDAURA MARTÍNEZ, M.J., "El sentido actual de la Ley Orgánica de Medidas de Protección Integral contra la Violencia de Género", Estudio Integral de la Violencia de Género: un análisis teórico-práctico desde el Derecho y las Ciencias Sociales, (dir. Martín, M.), Valencia, Tirant lo Blanch, 2018, pp. 137-164.

RINGROSE J., GILL R., LIVINGSTONE S., y HARVEY, L., A qualitative study of children, young people and 'sexting': report prepared for the NSPCC, 2012, http://www.nspcc.org.uk/Inform/resourcesforprofessionals/sexualabuse/sextingresearch-report_wdf89269.pdf 
SALA ORDOÑEZ, R., "L'estat embrionari de la violència de gènere digital", Món Jurídic: Revista de l'il·Lustre Col-Legi de l'advocacia de Barcelona, núm. 323 (junio-julio), 2019, pp. 20-21.

SANDELL, S., "Dating Apps, Rape and the Lack of Safety in Online Dating", HuffPost Blog, 24-02-2016.

SERRA CRISTOBAL, R., "Los derechos de la víctima en el proceso penal vs. medios de comunicación. Especial referencia a las víctimas de violencia por motivos de género", Revista española de derecho constitucional, Vol. 35, núm. 103, 2015, pp. 199-230.

STAUDE-MÜLLER, F., HANSEN, B. y VOSS, M., "How stressful is online victimization? Effects of victim's personality and properties of the incident", European Journal of Developmental Psychology, Vol. 9, núm. 2, 2012, pp. 260274.

VILLANUEVA-BLASCO, V.J. y SERRANO-BERNAL, S., "Patrón de uso de internet y control parental de redes sociales como predictor de sexting en adolescentes: una perspectiva de género", Revista de Psicología y Educación, Vol. 14, núm. 1, 2019, pp. 16-26.

WALKER, K., y SLEATH, E., "A systematic review of the current knowledge regarding revenge pornography and non-consensual sharing of sexually explicit media", Aggression and Violent Behavior, Vol. 36, 2017, pp. 9-24. 